\title{
Examination of the Clinical Queries and Systematic Review "hedges" in EMBASE and MEDLINE"
}

\author{
Susan M. Bradley ${ }^{2}$
}

\begin{abstract}
Introduction - This investigation sought to determine whether the methodological search filters in place as Clinical Queries limits in OvidSP EMBASE and OvidSP MEDLINE had been modified from those written by Haynes et al. [1] and whether the translations of these in PubMed and EBSCO MEDLINE were reliable. The translated National Library of Medicine (NLM) Systematic Reviews hedges in place in OvidSP MEDLINE and EBSCO MEDLINE were also examined. Methods - Search queries were run using the Clinical Queries and Systematic Reviews hedges incorporated into OvidSP EMBASE, OvidSP MEDLINE, PubMed, and EBSCO MEDLINE to determine the reliability of these limits in comparison with the published hedge search strings. Results - Five of the OvidSP EMBASE Clinical Queries hedges produced results that were different from the published search strings. Three of the EBSCO MEDLINE and five of the PubMed translated Clinical Queries hedges yielded markedly different results $(>10 \%$ difference) than those obtained using the OvidSP MEDLINE hedge counterparts. The OvidSP MEDLINE Systematic Reviews subject subset hedge was found to have a major error, which has been corrected. Discussion - Translations of hedges to appropriate syntax for other database platforms may result in significantly different search results. The platform searched should ideally be the one for which the hedges were written and tested. Regardless, the hedges in place may not be the same as the published hedge search strings. Quality control testing is needed to ensure that the hedges in place as limits are the same as those that have been published.
\end{abstract}

\section{Introduction}

Health sciences librarians are routinely involved in formulating focused questions and searching the literature for the best available evidence. Doing this requires a thorough knowledge of the major biomedical databases, the syntax of the particular platform being used, and the evidence-based medicine (EBM) limits and methodological search filters that are available in these databases.

The MEDLINE and EMBASE databases both contain very large numbers of records, the vast majority of which are not "clinically applicable" [1]. Methodological evidence-based search filters or "hedges" came about in the development of search techniques for clinically sound reports for various clinical topics and have become extremely important in biomedical searching. They have been referred to by Bachmann et al. as a "cornerstone in information retrieval in evidence-based practice" [2]. The term "methodological search filter" was defined by Wilczynski et al. as "a search term or terms...that select studies... at the most advanced stages of testing for clinical application" [3]. Alternate terms that have been used interchangeably include hedges, optimal search strategies, optimal search filters, search strategies, quality filters, search filters, or clinical queries [4].

Hedges written by the McMaster University Health Information Research Unit (HIRU) Hedges group (the Haynes group) [3,5-23] for the purpose of "improving the detection of studies of high quality for clinical practice" [1] have been incorporated into the Ovid EMBASE, Ovid MEDLINE, EBSCO MEDLINE, and PubMed database platforms as "Clinical Queries" hedges. These hedges were written for the clinical purpose categories diagnosis, therapy, etiology, prognosis, clinical prediction guides, and reviews [3]. They were developed empirically for Ovid MEDLINE and Ovid EMBASE by examining large sets of single or multiple search "terms" (e.g., Medical Subject Headings (MeSH), textwords, and publication types) that best expressed each clinical purpose category [3]. A gold standard set of journal articles for the year 2000 was developed and hand searched to identify the relevancy of articles for particular clinical purposes. By comparing the search results of the various hedge filters to the set already established as relevant, the sensitivity, selectivity, precision, and accuracies of the various hedges were determined at the time they were developed for the

S.M. Bradley. MLIS Candidate, School of Library, Archival and Information Studies, Irving K. Barber Learning Centre, 470-1961 East Mall, University of British Columbia, Vancouver, BC V6T 1Z1, Canada (sue.slais@gmail.com).

${ }^{1}$ This paper has been peer-reviewed.

${ }^{2}$ Susan M. Bradley received the Login Canada Student Paper Prize for this featured article. 
particular questions searched. The final hedges chosen for each clinical purpose category were those that met the conditions required for single or multiple terms with highest sensitivity, highest specificity, and minimal difference between sensitivity and specificity [3]. The Haynes hedges published for Ovid MEDLINE also had translations written for PubMed syntax [3].

The Systematic Reviews topic (or subject) subset filter [24] written by the U.S. National Library of Medicine (NLM) has been incorporated into PubMed, and translations of it are in use in OvidSP MEDLINE and EBSCO MEDLINE. The intent of this hedge is "to retrieve citations identified as systematic reviews, meta-analyses, reviews of clinical trials, evidence-based medicine, consensus development conferences, guidelines, and citations to articles from journals specializing in review studies of value to clinicians" [25]. It was developed based on research published by Shojania et al. in 2001 [26] and has gradually been modified over the years. However, it is pragmatic, and has not undergone testing for sensitivity, selectivity, precision, or accuracy [27].

The author of this paper noted that entering "equivalent" search strings in different MEDLINE interfaces and applying "equivalent" hedges sometimes led to extremely different search results. Although it would be expected that different interfaces to the same database would give equivalent search results to equivalent queries, studies carried out of searches run using different MEDLINE platforms have shown that this is not always the case [2830]. The Field Guides for Ovid MEDLINE and Ovid EMBASE state that the Clinical Queries limits are "based on the work of R. Brian Haynes MD, PhD et al. of the Health Information Research Unit (HIRU) at McMaster University" [25,31]. This could mean that these hedges are identical to those published by Haynes et al. or that modifications had been made. No studies could be found that examined whether or not the incorporated EBM hedges are indeed the same as those written by Haynes et al. or by the NLM. This investigation came about in an attempt to determine whether the relevant filters in place in the databases examined have been entered as originally written and whether the syntax translations written give equivalent results in different MEDLINE platforms. The specific hedges examined include the Clinical Queries hedges in OvidSP EMBASE, PubMed, OvidSP MEDLINE and EBSCO MEDLINE, and the NLM Systematic Reviews topic subset hedge built into PubMed, OvidSP MEDLINE, and EBSCO MEDLINE.

\section{Methods}

\section{Clinical queries hedges}

An examination was undertaken of the Clinical Queries hedges in place against the published hedge search strings for OvidSP EMBASE, OvidSP MEDLINE, and PubMed. Search results were compared both in terms of the number of hits and actual records retrieved. Identical search results contained identical records. In cases of differences in the records retrieved, percentage differences were calculated of the observed number of hits obtained using the Clinical
Queries limit versus those obtained using the published hedge search string.

Testing of the performance of Ovid MEDLINE Clinical Queries hedges translated to PubMed and EBSCO MEDLINE syntax was carried out. The percentage difference in number of hits for the second MEDLINE platform versus the OvidSP platform was calculated for various searches. Search results were classed as "markedly" different when the difference in number of hits was $>10 \%$. For such cases, modified search strings were written and tested. As there was no gold standard set for comparison, no calculations of sensitivity, selectivity, precision, or accuracy could be made of the results for these searches.

\section{Systematic reviews hedges}

Examinations of the Haynes Clinical Queries "specific" Review hedges in OvidSP EMBASE and MEDLINE, EBSCO MEDLINE, and PubMed were carried out. The Cochrane Database of Systematic Reviews was used as a simple gold standard set to compare percentage search results.

A comparison was also made of the results found using the NLM Systematic Reviews subject subset hedges in OvidSP MEDLINE and EBSCO MEDLINE compared with the retrieval found using PubMed.

\section{Results}

Part 1. Comparisons of PubMed, OvidSP MEDLINE, and EBSCO MEDLINE search syntax

To be able to compare and translate filters written for Ovid MEDLINE or PubMed, a comparison was made of the syntaxes by which search strings involving search fields or MeSH headings were entered for each database. EBSCO MEDLINE was also examined to some degree. A comparison of the entry syntaxes and results of some "equivalent" keyword and MeSH searches carried out using these three MEDLINE interfaces is presented in Tables 1A, 1B, and 1C.

Truncated entry terms in PubMed were found to give different results than equivalent truncated terms in Ovid MEDLINE. In PubMed, stopwords are searched for if a phrase is in quotation marks or is truncated. Ovid MEDLINE no longer has stopwords [32-34]. As a result, direct syntax translations of entry terms involving truncation or stopwords can yield different results between PubMed and Ovid MEDLINE.

\section{Duplicate OvidSP MEDLINE records}

Searches were carried out of all articles in the journal Neurologist. The expectation was that for the terms entered, EBSCO MEDLINE and Ovid MEDLINE(R) In-Process \& Other Non-Indexed Citations and Ovid MEDLINE(R) would produce identical or fewer hits than PubMed. Instances of fewer hits would be explained as recent entries that had not yet been uploaded. This was found to be true for EBSCO MEDLINE. However, Ovid MEDLINE(R) In-Process \& Other Non-Indexed Citations and Ovid MEDLINE(R) produced more hits than PubMed. An investigation of this discrepancy led to the discovery of duplicate Ovid MEDLINE entries. Ovid 
Table 1. Comparison of entry syntaxes for OvidSP MEDLINE, PubMed, and EBSCO MEDLINE.

(A) Comparison of search string syntaxes for OvidSP MEDLINE, PubMed, and EBSCO MEDLINE (as of 20 July 2009)

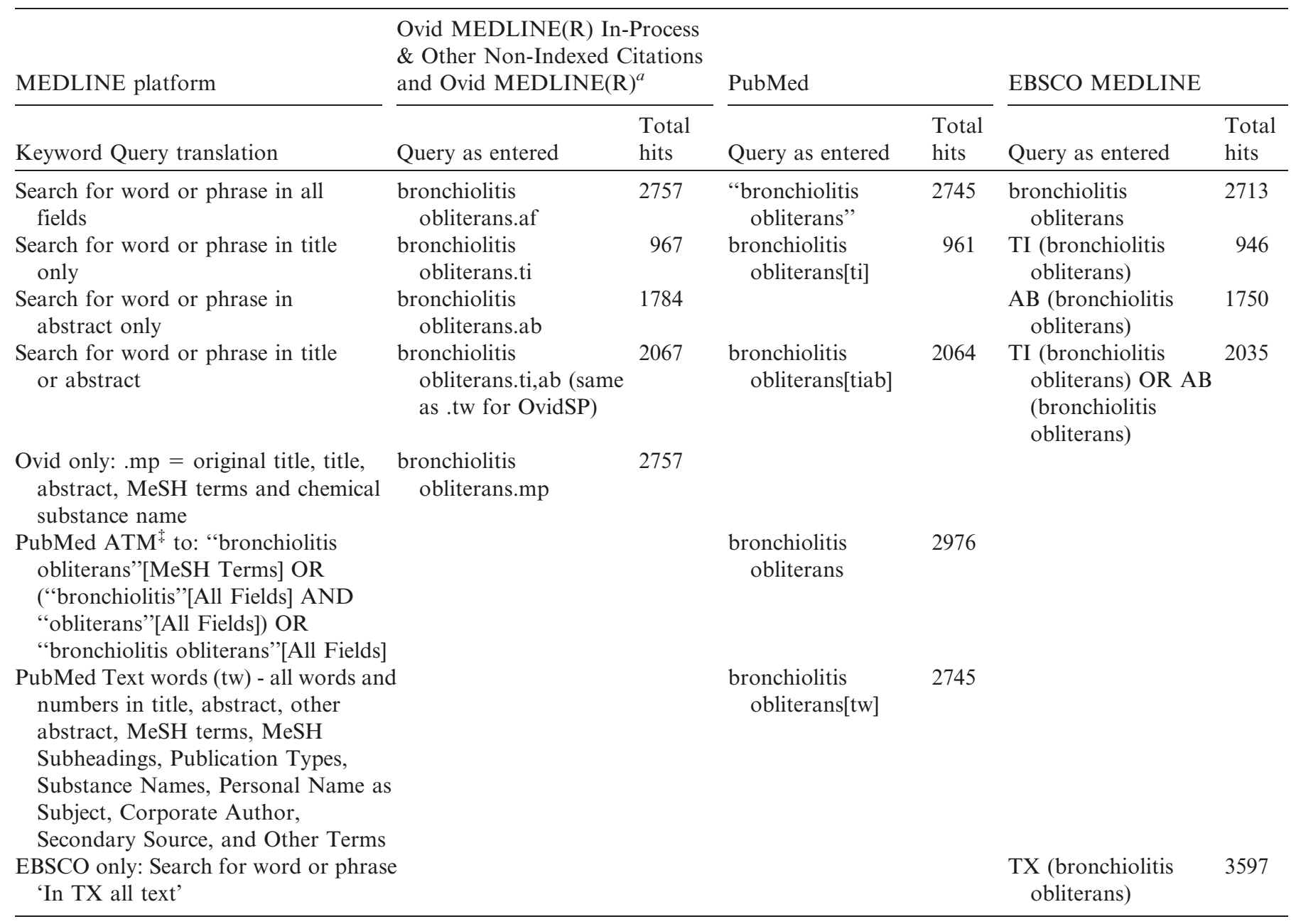

(B) Comparison of MeSH heading search string syntaxes for OvidSP MEDLINE, PubMed, and EBSCO MEDLINE (as of 20 July 2009)

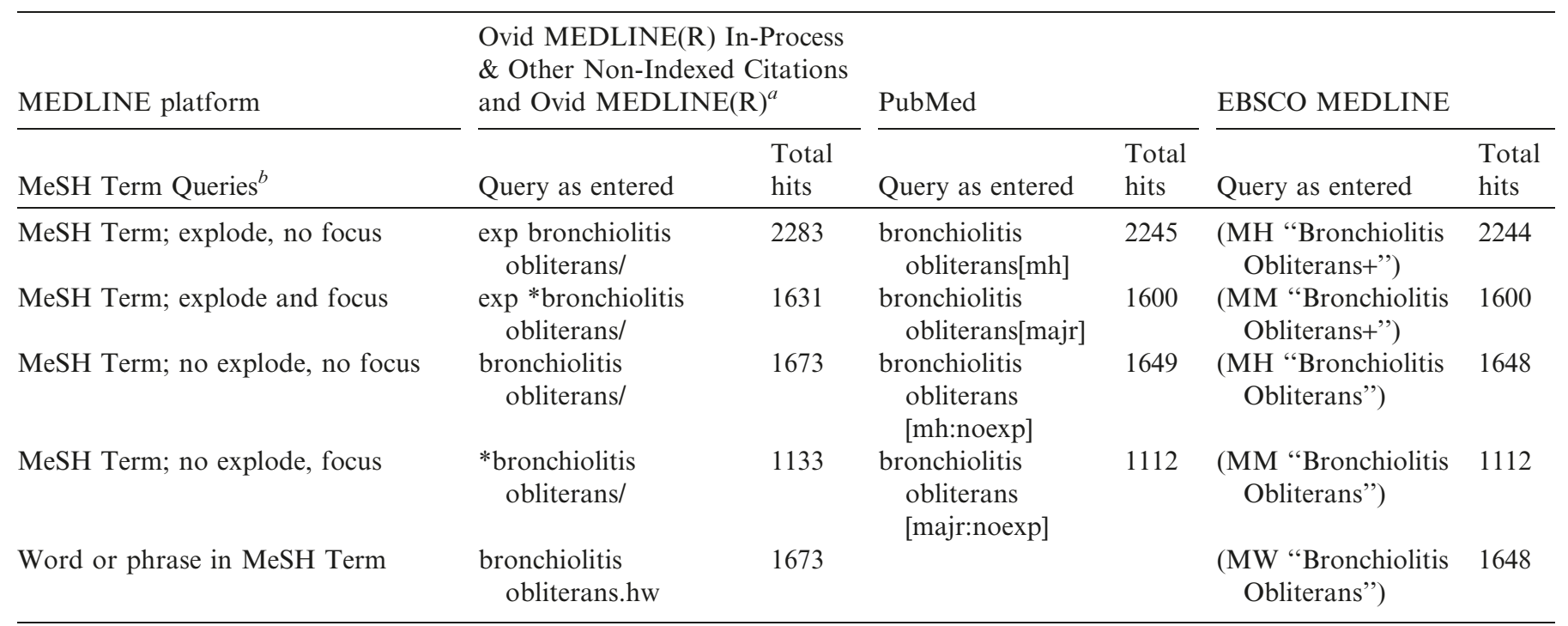


Table 1. (continued).

(C) Comparison of MeSH Heading plus SubHeading search string syntaxes for PubMed and OvidSP MEDLINE

\begin{tabular}{|c|c|c|c|c|}
\hline $\begin{array}{l}\text { MeSH Heading plus } \\
\text { Subheading Queries }^{c}\end{array}$ & $\begin{array}{l}\text { PubMed search } \\
\text { string entered }\end{array}$ & Total hits & $\begin{array}{l}\text { OvidSP MEDLINE search } \\
\text { string entered }\end{array}$ & Total hits \\
\hline MeSH Subheading (exploded) & diagnosis[sh] & 3455712 & di.xs & 3525142 \\
\hline $\begin{array}{l}\text { MeSH Term (exploded) AND } \\
\text { Subheading (not exploded) }\end{array}$ & $\begin{array}{l}\text { Respiratory Tract Infection[mh] } \\
\text { AND therapy[sh:noexp] }\end{array}$ & 25685 & $\begin{array}{l}\text { exp Respiratory Tract } \\
\text { Infection/ AND th.fs }\end{array}$ & 25122 \\
\hline $\begin{array}{l}\text { MeSH Term (exploded) AND } \\
\text { Subheading (exploded) }\end{array}$ & $\begin{array}{l}\text { Respiratory Tract Infection[mh] } \\
\text { AND therapy[sh] }\end{array}$ & 110240 & $\begin{array}{l}\text { exp Respiratory Tract } \\
\text { Infection/ AND th.xs }\end{array}$ & 111936 \\
\hline $\begin{array}{l}\text { MeSH Term (exploded)/Subheading } \\
\text { (not exploded) }\end{array}$ & & & $\begin{array}{l}\text { exp Respiratory Tract } \\
\text { Infection/th }\end{array}$ & 19172 \\
\hline $\begin{array}{l}\text { MeSH Term (not exploded)/ } \\
\text { Subheading(exploded) }\end{array}$ & $\begin{array}{l}\text { Respiratory Tract } \\
\text { Infection[mh:noexp]/th }\end{array}$ & 12473 & & \\
\hline $\begin{array}{l}\text { MeSH Term (not exploded)/ } \\
\text { Subheading (not exploded) }\end{array}$ & $\begin{array}{l}\text { Respiratory Tract Infection/ } \\
\text { th[mh:noexp] }\end{array}$ & 1192 & $\begin{array}{l}\text { Respiratory Tract } \\
\text { Infection/th }\end{array}$ & 1232 \\
\hline
\end{tabular}

MEDLINE now creates a new record when revisions are made to an existing record. Both the original and the revised records have the same unique identifier. This practice of creating an additional record when a revision is made appears to have begun in late 2008. It is not unusual for an OvidSP MEDLINE search of a MeSH term to produce up to $2.5 \%$ more hits than the equivalent search in PubMed. After de-duplicating ("deduping") these particular search results and determining the number of unique entries, OvidSP MEDLINE, PubMed, and EBSCO MEDLINE were found to produce the same number of records. An obvious implication of this finding is that one must be careful to delete the older of two "duplicate" entries when deduping such OvidSP MEDLINE search results.

In the case of the OvidSP EMBASE searches carried out, no instances of duplicate records were found. The issue of duplicate Ovid EMBASE records has been previously reported [35], and this also came about when record revisions were made. In the search example reported, 14 duplicates were found having the same accession number, in a total of 1686 hits (0.8\%). Rerunning accession numbers reported as duplicates found there to now be only one record for each. This problem may have been fixed in OvidSP EMBASE.

\section{Part 2. Clinical queries hedges}

Both Ovid EMBASE and MEDLINE have added some of the Haynes Clinical Queries hedges [3,5-23] to their limits. Those not built in can be used by entering the published hedge search string.

The Ovid MEDLINE hedges developed were translated in syntax to be used in PubMed [3]. The PubMed Clinical Queries filter table [27] details these hedges. However, the statistics listed by the NLM are those determined for the Ovid MEDLINE syntax. Given the problems found with translations of Ovid MEDLINE and PubMed search string syntax, quoting the same statistics for the PubMed filters is likely misleading in some cases.

The reliability of the EBSCO MEDLINE translations of the Haynes Clinical Queries hedges was also investigated.

\section{OvidSP EMBASE}

The only EMBASE platform examined was OvidSP. For this reason, the comparison done was of the results of a search query (EMTREE heading) together with each Clinical Queries limit in place and with the actual hedge search strings published by Haynes et al. [5-12]. Full results are summarized in Appendix A in this paper's Supplementary data. ${ }^{3}$

Of the 33 Clinical Queries hedges examined, 27 gave identical search results to those obtained by applying the Haynes hedge. In one case (Qualitative studies - 1 term min difference) the search string could not be run as the EMTREE heading it consisted of was not recognized. The remaining five limits gave results that differed from those found using the Haynes hedge strings (Table 2). For the particular search run, percentage differences ranged from $-6.0 \%$ to $86.9 \%$.

OvidSP MEDLINE, PubMed, and EBSCO MEDLINE

OvidSP MEDLINE, PubMed, and EBSCO MEDLINE use versions of the Haynes Clinical Queries hedges. These limit titles, together with the search strings that correspond to the Haynes hedges and the translations to PubMed syntax are summarized in Appendix B in this paper's Supplementary data. ${ }^{3}$ The search strings used in EBSCO MEDLINE were never determined.

A comparison was made of the search results obtained using the 27 OvidSP MEDLINE Clinical Queries limits versus the search strings of the Haynes hedges. All of these

\footnotetext{
${ }^{3}$ Appendices A, B, and C are available as Supplementary data on the Web at http://pubservices.nrc-cnrc.ca/jchla.
} 
Table 2. Discrepancies found in OvidSP EMBASE hedge search results.

\begin{tabular}{|c|c|c|c|c|c|}
\hline Haynes EMBASE hedge title & $\begin{array}{l}\text { Haynes EMBASE hedge search } \\
\text { string }\end{array}$ & Hits & EMBASE limit title & Hits & $\begin{array}{l}\% \\
\text { difference }\end{array}$ \\
\hline $\begin{array}{l}\text { Diagnosis ( } 2 \text { or more terms min } \\
\text { difference) }\end{array}$ & $\begin{array}{l}\text { sensitive*.tw. OR diagnostic } \\
\text { accuracy.sh. OR diagnostic.tw. }\end{array}$ & 7919 & Diagnosis (optimized) & 10120 & 27.8 \\
\hline $\begin{array}{l}\text { Reviews ( } 2 \text { or more terms, best } \\
\text { optimization) }\end{array}$ & $\begin{array}{l}\text { meta-analysis*.mp. } \\
\text { OR search*.tw. } \\
\text { OR review.pt. }\end{array}$ & 20275 & $\begin{array}{l}\text { Reviews ( } 2 \text { or more terms } \\
\text { min difference) }\end{array}$ & 20331 & 0.3 \\
\hline $\begin{array}{l}\text { Qualitative studies ( } 2 \text { or more } \\
\text { terms, best optimization) }\end{array}$ & $\begin{array}{l}\text { interview*.tw. OR exp health } \\
\text { care organization/ } \\
\text { OR experiences.tw. }\end{array}$ & 6403 & $\begin{array}{l}\text { Qualitative studies ( } 2 \text { or } \\
\text { more terms min difference) }\end{array}$ & 6017 & -6.0 \\
\hline $\begin{array}{l}\text { Treatment ( } 2 \text { or more terms } \\
\text { high specificity) }\end{array}$ & $\begin{array}{l}\text { double-blind*.mp. } \\
\text { OR placebo*.tw. } \\
\text { OR blind*.tw. }\end{array}$ & 5741 & $\begin{array}{l}\text { Treatment ( } 2 \text { or more terms } \\
\text { high specificity) }\end{array}$ & 10728 & 86.9 \\
\hline
\end{tabular}

Note: Search: exp heart infarction/ with limit applied, or ANDed to search string results. Run 29 June 2009 (1980 - 2009 week 26 ).

OvidSP MEDLINE hedges gave identical results to those of the " 2 or more term" Haynes hedges. None of the hedges in place as limits were found to have been modified from the published search strings.

Comparisons of results obtained for identical MeSH term searches with the Clinical Queries hedges were made for OvidSP and EBSCO MEDLINE, and PubMed (MeSH search terms "Myocardial infarction"; "Neoplasms"; and "Diabetes Mellitus, Type 2"; with no explode or focus). The full results are summarized in Appendix $\mathrm{C}$ in this paper's Supplementary data. ${ }^{3}$ If the hedge translations were exact, it would be expected that PubMed and EBSCO MEDLINE would produce identical results to OvidSP MEDLINE, with the exception of fewer hits for records not yet uploaded. Although discrepancies in the number of hits would indicate a difference in the search results, the same number of hits would not necessarily mean identical search results. For the MeSH terms themselves with no limits applied, OvidSP MEDLINE produced more hits as a result of duplicate records now being produced when record revisions are made. Deduping was not feasible given the size of the datasets. This duplicate record problem meant that for identical results there would be an expected negative percent difference for comparisons made to the OvidSP MEDLINE search result.

Although the majority of searches with hedge limits applied yielded "similar" numbers of records, there were some obvious discrepancies (Table 3). For PubMed versus OvidSP MEDLINE, there were five Clinical Queries hedges that gave markedly different results $(>10 \%$ difference in number of hits). These were the Haynes Clinical Queries hedges rewritten for PubMed syntax for the following:

- Therapy (2 or more terms min difference) - too narrow

- Causation (Etiology) (2 or more terms high sensitivity) too broad

- Causation (Etiology) (2 or more terms high specificity) too broad
- Causation (Etiology) (2 or more terms min difference) too narrow

- Economics (2 or more terms min difference) - too narrow

Hedges found to yield markedly fewer results are labelled as "too narrow", whereas those giving markedly more have been labelled "too broad". Only the Causation/Etiology sensitivity and Causation/Etiology specificity hedges were built into PubMed. Three of these five hedges involved truncated search terms that led to a message indicating that only the first 600 variations were used and that lengthening of the root word would be required to search for all endings. The search results differences show that the publication by the NLM of the percentage sensitivity, specificity, precision, and accuracy for the PubMed Clinical Queries hedges being the same as for the Ovid MEDLINE hedges $[3,27]$ is misleading and, in some cases, incorrect.

PubMed hedge syntax translations were examined, and modifications of the five hedges giving anomalous results were written. The general logic taken is that PubMed searches that yielded results that were too narrow and involved the use of a title/abstract field might produce "better" results by searching as a textword, and vice versa for too broad results. Those involving a truncated term might be better done by removing the truncation and instead using the reasonable terms that one might expect to encounter. The comparative search results using the modified versus published hedges are outlined in Table 4 . Results reported are of percentage difference in number of hits. Although the modified hedges may appear improved, only a careful study using a gold standard set would allow this to be determined.

In the case of the "Therapy ( 2 or more terms min difference)" hedge, the search results were too narrow. Broadening the search syntax by changing the title/ abstract keyword entries to textword and also adding the British spelling of randomized (randomised) led to more equivalent search results. 
Table 3. Discrepancies found between PubMed and OvidSP MEDLINE Clinical Queries search results (searches run 12 July 2009).

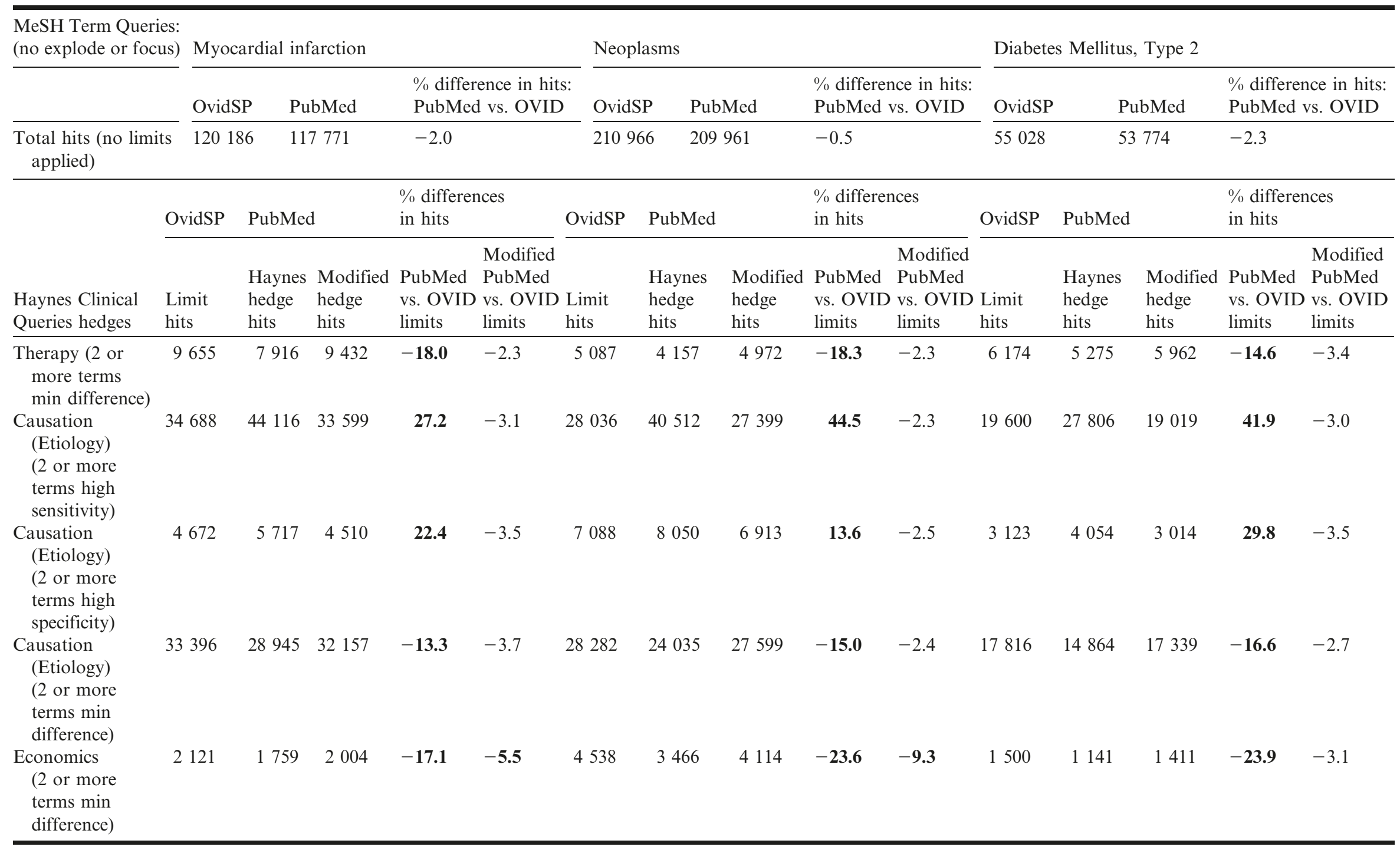


The Causation (Etiology) (2 or more terms high sensitivity) hedge was found to be too broad. This one involves the use of a truncated term. The original Ovid filter uses the textword "between group*", but for PubMed this was rewritten as "group*[tw]". In PubMed, "between" is a stopword, and this is presumably why it was dropped from the translated search string. However, by including it as part of a truncated phrase, this stopword is automatically included [32,33]. In addition, having group* entered as a textword in Ovid narrows the field to title, abstract. To translate literally, it is proposed to have this term changed to "between group*[tiab]" in PubMed.

Three EBSCO MEDLINE Clinical Queries hedges gave markedly different results from those obtained using OvidSP MEDLINE. These were the Haynes hedges rewritten for EBSCO MEDLINE syntax for the following:

- Clinical prediction guides (2 or more terms high specificity) - too narrow

- Clinical prediction guides (2 or more terms min difference) - too broad

- Causation (Etiology) (2 or more terms high sensitivity) too broad

The Ovid MEDLINE syntax for the Clinical prediction guides ( 2 or more terms high specificity) hedge was "validation.tw. OR validate.tw". Translating this to EBSCO syntax as "TI validation $O R A B$ validation $O R$ TI validate OR AB validate" gave equivalent EBSCO MEDLINE search results to those found using PubMed.

\section{Part 3. Systematic review hedges}

An introduction to systematic reviews and a summary of their role in EBM can be found in Cook et al. [36]. Although systematic reviews are generally viewed as a publication type, neither EMBASE nor MEDLINE include a publication type for "systematic reviews". MEDLINE does include publication type "metaanalysis", but a systematic review can be done without including a meta-analysis, and a meta-analysis can be done of data published in a narrative literature review and thus not constitute a systematic review. An examination of the Systematic Review hedges available in OvidSP EMBASE and in OvidSP MEDLINE, EBSCO MEDLINE, and PubMed was undertaken.

\section{OvidSP EMBASE}

In EMBASE, there is an EMTREE indexing term for "systematic review". The reliability of using this indexing term was tested by using the Cochrane Database of Systematic Reviews as a simple gold standard set. Combining the search terms "Cochrane Database of Systematic Reviews.jn" AND "systematic review/" led to only $41 \%$ of these articles being retrieved.

The hedge used for systematic reviews in OvidSP EMBASE is the Haynes et al. "2 or more terms - best specificity" Clinical Queries Review hedge, which was tested in Part 2. Although the term "systematic" is not part of the title, the search string "meta-analysis.tw. OR systematic review.tw." reveals that it was written to include high quality evidence-based reviews. The " 2 or more terms, best sensitivity" hedge is targeted for all review articles. The Cochrane Database of Systematic Reviews was used as a simple gold standard set to test these Review hedges. The sensitive filter retrieved $100 \%$ of these records; the optimized filter retrieved all but one. For it, the publication type had been indexed as a "note", which explains its omission. Despite having been written to target systematic reviews and meta-analyses, the specific filter retrieved only $16 \%$ of eligible articles - less than the indexing term "systematic review". The hedges developed for EMBASE were developed and tested using a 55journal subset that did not include the Cochrane Database of Systematic Reviews [5].

\section{PubMed, OvidSP MEDLINE, and EBSCO MEDLINE Clinical queries "specific review" hedges}

In the PubMed, OvidSP MEDLINE, and EBSCO MEDLINE databases, the Haynes Clinical Queries "specific" Review hedges can be used to search for systematic reviews. As was done for EMBASE, the Clinical Queries Review hedges were compared in searching for "Cochrane Database of Systematic Reviews" articles.

In OvidSP MEDLINE the sensitive and optimized "Review" hedges found all but one article, which was indexed as publication type "Retraction of Publication". The specific hedge found $83 \%$ of the records, which was more efficient than its EMBASE counterpart. In addition, the hedges for MEDLINE were developed and tested using a dataset from 161 clinical journals, which did include the "Cochrane Database of Systematic Reviews" [37].

Both the PubMed and the EBSCO MEDLINE sensitive, selective, and optimized Haynes Clinical Queries Review hedges gave the same results as the corresponding OvidSP MEDLINE hedges (de-duped results) for the "Cochrane Database of Systematic Reviews" articles search.

\section{Systematic reviews subject subset hedges}

The MEDLINE databases examined all include a "Systematic Review" hedge in the Topic or Subject Subset $[38,39]$ limit section. This sounds as if this hedge is limiting the search to the subject of "systematic review" rather than the publication type. The PubMed Web site adds to the confusion by having this particular hedge also accessible by clicking on the sidebar Clinical Queries link, which implies it is one of the Haynes hedges. From this it appears that there are two different NLM Systematic Review hedges when there is only one. This particular hedge will now be examined.

OvidSP MEDLINE and EBSCO MEDLINE both have limits in place with their translations of this NLM hedge. In April 2009, a comparison of searches done using EBSCO MEDLINE versus PubMed yielded identical record lists. However, a major discrepancy was found between OvidSP MEDLINE and PubMed searches. On applying this hedge to OvidSP MEDLINE for the Cochrane Database gold standard set, it was discovered that the hedge was missing $40 \%$ of eligible records. 
Table 4. PubMed Clinical Queries run using proposed modified hedges.

\begin{tabular}{|c|c|c|c|c|}
\hline Optimized for & OvidSP syntax & Published PubMed translation & $\begin{array}{l}\text { Published PubMed translation } \\
\text { (short form) }\end{array}$ & $\begin{array}{l}\text { Proposed modified } \\
\text { PubMed syntax }\end{array}$ \\
\hline \multicolumn{5}{|l|}{ Therapy } \\
\hline \multicolumn{5}{|l|}{ Etiology } \\
\hline $\begin{array}{l}\text { Sensitive/ broad } \\
\text { (too broad) }\end{array}$ & $\begin{array}{l}\text { risk*.mp. OR exp }^{*} \text {. } \\
\text { cohort studies OR } \\
\text { between group*.tw. }\end{array}$ & $\begin{array}{l}\text { (risk*[Title/Abstract] OR } \\
\text { risk*[MeSH:noexp] OR risk } \\
\text { *[MeSH:noexp] OR cohort } \\
\text { studies[MeSH Terms] OR } \\
\text { group*[Text Word]) }\end{array}$ & $\begin{array}{l}\text { risk } *[\text { tiab }] \text { OR risk } *[\text { mh:noexp] } \\
\text { OR risk } *[\text { mh:noexp] OR } \\
\text { cohort studies[mh] OR } \\
\text { group*[tw] }\end{array}$ & $\begin{array}{l}\text { risk*[tiab] OR } \\
\text { risk*[mh:noexp] OR risk } \\
\text { *[mh:noexp] OR cohort } \\
\text { studies[mh] OR between } \\
\text { group*[tiab] }\end{array}$ \\
\hline $\begin{array}{l}\text { Specific/ narrow }{ }^{a} \\
\text { (too broad) }\end{array}$ & $\begin{array}{l}\text { relative risk*.tw. OR } \\
\text { risks.tw. OR cohort } \\
\text { stud*.mp. }\end{array}$ & $\begin{array}{l}\text { ((relative[Title/Abstract] AND } \\
\text { risk*[Title/Abstract]) OR } \\
\text { (relative risk[Text Word]) OR } \\
\text { risks[Text Word] OR cohort } \\
\text { studies[MeSH:noexp] OR } \\
\text { (cohort[Title/Abstract] AND } \\
\text { stud*[Title/Abstract])) }\end{array}$ & $\begin{array}{l}\text { (relative[tiab] AND risk*[tiab]) } \\
\text { OR (relative risk[tw]) OR } \\
\text { risks[tw] OR cohort } \\
\text { studies[mh:noexp] OR } \\
\text { (cohort[tiab] AND } \\
\text { stud*[tiab]) }\end{array}$ & $\begin{array}{l}\text { relative risk*[tiab] OR } \\
\text { risks[tw] OR cohort } \\
\text { stud*[tw] }^{*}\end{array}$ \\
\hline $\begin{array}{l}\text { Min difference } \\
\text { (too narrow) }\end{array}$ & $\begin{array}{l}\text { risk.mp. OR } \\
\text { mortality.mp. OR } \\
\text { cohort.tw. }\end{array}$ & $\begin{array}{l}\text { risk[Title/Abstract] OR } \\
\text { risk[MeSH:noexp] OR } \\
\text { mortality[Title/Abstract] OR } \\
\text { mortality[MeSH:noexp] OR } \\
\text { cohort[Title/Abstract] }\end{array}$ & $\begin{array}{l}\text { risk[tiab] OR risk[mh:noexp] } \\
\text { OR mortality[tiab] OR } \\
\text { mortality[mh:noexp] OR } \\
\text { cohort[tiab] }\end{array}$ & $\begin{array}{l}\text { risk[tw] OR mortality[tiab] } \\
\text { OR } \\
\text { mortality[mh:noexp] } \\
\text { OR cohort[tiab] }\end{array}$ \\
\hline \multicolumn{5}{|l|}{ Economics } \\
\hline $\begin{array}{l}\text { Min difference } \\
\quad \text { (too narrow) }\end{array}$ & $\begin{array}{l}\text { cost*.mp. OR cost }^{*} \text { ORefit analys*.mp. } \\
\text { benefilth care } \\
\text { OR health } \\
\text { costs.mp. }\end{array}$ & $\begin{array}{l}\text { cost*[Title/Abstract] OR “costs } \\
\text { and cost } \\
\text { analysis”[MeSH:noexp] OR } \\
\text { cost benefit analys*[Title/ } \\
\text { Abstract] OR cost-benefit } \\
\text { analysis[MeSH Term] OR } \\
\text { health care costs[MeSH:noexp] }\end{array}$ & $\begin{array}{l}\text { cost*[tiab]OR “costs and cost } \\
\text { analysis”[mh:noexp] OR cost } \\
\text { benefit analys*[tiab] OR cost- } \\
\text { benefit analysis[mh] OR } \\
\text { health care costs[mh:noexp] }\end{array}$ & $\begin{array}{l}\text { cost [tw] OR costs[tw] OR } \\
\text { "costs and cost } \\
\text { - analysis"[mh:noexp] OR } \\
\text { cost benefit analys*[tw] } \\
\text { OR health care costs[tw] }\end{array}$ \\
\hline
\end{tabular}

${ }^{a}$ These entries involved truncated search strings that led to a message indicating that only the first 600 variations were used and that lengthening of the root word would be required to search for all endings.

\begin{tabular}{ll}
\hline Search & Results \\
\hline 1. Cochrane database of systematic reviews.jn & 5803 \\
2. Limit 1 to systematic reviews & 3457 \\
\hline
\end{tabular}

On reviewing the NLM hedge, it was not clear why all Cochrane Database articles were not retrieved. The OvidSP MEDLINE hedge had not yet been updated to the most recent version, but even the original one published by Shojania et al. [26] would have retrieved all of these records.

In an e-mail from the author, Ovid Customer Service was asked to supply their version of the NLM hedge. Initially the response was that "other search statements" had been added by Ovid but that the information was proprietary and would not be released. The full search string of the filter was subsequently provided to the author by e-mail, on the condition that it only be used for the course report being written [40].

An evaluation of the search string provided by Ovid found that it did not lead to any of the anomalous exclusions noted above; they were due to a database error. Two other NLM subject subset hedges were chosen at random (AIDS and "space life sciences") and search results obtained in OvidSP MEDLINE compared with PubMed. Major discrepancies were found for all three of these subject subset filters (Table 5). Ovid Customer Service was notified of the problem, and an investigation was undertaken. As of 23 June 2009, OVID Customer Service reported to the author by e-mail that these three OvidSP MEDLINE hedges had been fixed.

\section{Discussion}

This study began with the simple question: Why do "equivalent" search strings and "equivalent" hedges used in different interfaces to equivalent databases sometimes lead to very different search results? The specific hedges examined included the Clinical Queries hedges in OvidSP EMBASE and MEDLINE, PubMed and EBSCO MEDLINE, and the NLM Systematic Reviews subject subset hedge built into PubMed, OvidSP MEDLINE, and EBSCO 
Table 5. Queries run using three subject subset hedges in PubMed and OvidSP MEDLINE (run in April 2009 and then in June 2009).

\begin{tabular}{|c|c|c|c|c|c|c|}
\hline Database query & PubMed & $\begin{array}{l}\text { OvidSP } \\
\text { MEDLINE }\end{array}$ & $\begin{array}{l}\% \text { difference OVID } \\
\text { vs. PubMed }\end{array}$ & PubMed & $\begin{array}{l}\text { OvidSP } \\
\text { MEDLINE }\end{array}$ & $\begin{array}{l}\% \text { difference } \\
\text { OVID vs. PubMed }\end{array}$ \\
\hline Date run & 15 April 2009 & & & 24 June 2009 & & \\
\hline Limit 1 to systematic reviews & 64 & 44 & -45.5 & 65 & 65 & 0 \\
\hline Date run & 13 April 2009 & & & 23 June 2009 & & \\
\hline Exp orthostatic intolerance/ & 5372 & 5408 & 0.7 & 5408 & 5465 & 1.0 \\
\hline Limit 1 to space life sciences & 3265 & 1260 & -159.1 & 3281 & 3336 & 1.6 \\
\hline
\end{tabular}

MEDLINE. No reports of hedge modifications or errors were found in the literature that would explain the observed discrepancies. Syntax issues were examined to ensure that the search strings entered in the different databases being compared were "equivalent". A number of differences were found that led to problems in syntax translations between databases, and these were summarized in Table 1.

In the case of OvidSP EMBASE, 27 of the 33 Clinical Queries limits in place were found to give identical results to the search strings published by Haynes et al. One could not be run because the EMTREE heading was not recognized. Another five gave search results that differed from those obtained using the Haynes hedge search strings. The absolute percentage difference in numbers of search results ranged from minor $(0.3 \%)$ to major $(87 \%)$. For these, the search strings of the limits in place have been modified from the published search strings. It is not known if this was done intentionally or in error.

The OvidSP MEDLINE Clinical Queries limits were all found to give identical results to the hedges published by Haynes et al. The Clinical Queries hedges in place in PubMed or published by Haynes et al. for PubMed [3] are translations of the original Ovid syntax. There are search syntax differences between PubMed and Ovid MEDLINE, and this leads to translation problems in many cases. For five hedges, results were markedly different $(>10 \%$ difference). Proposed modifications to these translations have been made, although their sensitivity, specificity, precision, and accuracy cannot be determined without comparing search results with those of a hand search of a gold standard set. Although they may appear to be a better translation based on numbers of "hits", this is yet to be proven.

EBSCO MEDLINE has Clinical Queries filters in place that are obviously based on the Haynes hedges. Three of these were found to give markedly different results from OvidSP MEDLINE. A translation was written for one of these hedges, and it was found to give equivalent results to its PubMed counterpart.

The NLM Systematic Reviews subject subset hedge searches for high quality EBM material such as systematic reviews, clinical practice guidelines, and consensus development conferences. Although it has not undergone testing to determine sensitivity, specificity, precision, and accuracy, translations of it are available in OvidSP and EBSCO MEDLINE. A major problem was found with the OvidSP
MEDLINE version of this, as well as with two other subject subset filters. Ovid was notified, and the problem was resolved in late June 2009. Thereafter, similar search results were found using the "equivalent" hedges in the three MEDLINE interfaces examined. The fact that Ovid MEDLINE subject subset filter search strings contain proprietary information that is not publicly available is alarming. Health science librarians need to be able to evaluate hedges to determine whether or not to use them. Without being able to examine the search string, it is difficult to evaluate the possible utility or limitations of a filter.

\section{Conclusions}

Significant problems were found with the translations of Ovid MEDLINE Clinical Queries hedges to other platforms. Therefore, Ovid MEDLINE should be the platform used for these particular hedges, if one has access to it. Conversely, the NLM Systematic Reviews subject subset hedge was written for PubMed; the OvidSP "equivalent" was out of date and had a serious problem that has been corrected. Search strings used for subject subsets in OvidSP MEDLINE may contain "proprietary information" and therefore not be publicly available; further, the dates of the latest revisions are not readily available. For these reasons, it would be best to use PubMed for Systematic Review and other subject subset hedge searches.

\section{Acknowledgments}

The author would like to thank Dean Giustini, University of British Columbia biomedical branch librarian, for his guidance and encouragement throughout the course of this project.

\section{References}

1. Haynes RB, Wilczynski N, McKibbon KA, Walker CJ, Sinclair JC. Developing optimal search strategies for detecting clinically sound studies in MEDLINE. J Am Med Inform Assoc. 1994 Nov-Dec;1(6):447-58.

2. Bachmann LM, Coray R, Estermann P, Ter Riet G. Identifying diagnostic studies in MEDLINE: reducing the number needed to read. $J$ Am Med Inform Assoc. 2002 NovDec;9(6):653-8. 
3. Wilczynski NL, Walker CJ, McKibbon KA, Haynes RB. Reasons for the loss of sensitivity and specificity of methodologic MeSH terms and textwords in MEDLINE. Proc Annu Symp Comput Appl Med Care. 1995:436-40.

4. Jenkins M, Johnson F. Awareness, use and opinions of methodological search filters used for the retrieval of evidence-based medical literature-a questionnaire survey. Health Info Libr J. 2004 Mar;21(1):33-43. doi:10.1111/ j.1471-1842.2004.00480.x.

5. Health Information Research Unit, McMaster University. Search strategies for EMBASE in Ovid Syntax [Internet]. June 2007. [rev. 29 Jun 2007; cited 8 Sep 2009]. Available from: http:// hiru.mcmaster.ca/hiru/HIRU_Hedges_EMBASE_Strategies. aspx.

-6. Wilczynski NL, Haynes RB. EMBASE search strategies for identifying methodologically sound diagnostic studies for use by clinicians and researchers. BMC Med. 2005 Mar 29;3:7. doi:10.1186/1741-7015-3-7.

7. Wilczynski NL, Haynes RB. Optimal search strategies for detecting clinically sound prognostic studies in EMBASE: an analytic survey. J Am Med Inform Assoc. 2005 Jul-Aug; 12(4):481-5. doi:10.1197/jamia.M1752.

8. Haynes RB, Kastner M, Wilczynski NL, Hedges Team. Developing optimal search strategies for detecting clinically sound and relevant causation studies in EMBASE. BMC Med Inform Decis Mak. 2005 Mar 22;5:8. doi:10.1186/14726947-5-8.

-9. Holland JL, Wilczynski NL, Haynes RB, Hedges Team. Optimal search strategies for identifying sound clinical prediction studies in EMBASE. BMC Med Inform Decis Mak. 2005 Apr 29;5:11. doi:10.1186/1472-6947-5-11.

10. Wong SS, Wilczynski NL, Haynes RB. Developing optimal search strategies for detecting clinically sound treatment studies in EMBASE. J Med Libr Assoc. 2006 Jan;94(1):41-7.

11. Wilczynski NL, Haynes RB, Hedges Team. EMBASE search strategies achieved high sensitivity and specificity for retrieving methodologically sound systematic reviews. J Clin Epidemiol. 2007 Jan;60(1):29-33. doi:10.1016/j.jclinepi.2006. 04.001 .

-12. McKinlay RJ, Wilczynski NL, Haynes RB, Hedges Team. Optimal search strategies for detecting cost and economic studies in EMBASE. BMC Health Serv Res. 2006 Jun 6;6:67. doi:10.1186/1472-6963-6-67.

13. Walters LA, Wilczynski NL, Haynes RB, Hedges Team. Developing optimal search strategies for retrieving clinically relevant qualitative studies in EMBASE. Qual Health Res. 2006 Jan;16(1):162-168. doi:10.1177/1049732305284027.

14. Wong SS, Wilczynski NL, Haynes RB. Comparison of topperforming search strategies for detecting clinically sound treatment studies and systematic reviews in MEDLINE and EMBASE. J Med Libr Assoc. 2006 Oct;94(4):451-5.

15. Wilczynski NL, McKibbon KA, Haynes RB. Enhancing retrieval of best evidence for health care from bibliographic databases: calibration of the hand search of the literature. Stud Health Technol Inform. 2001;84(Pt 1):390-3.

16. Wong SS, Wilczynski NL, Haynes RB, Ramkissoonsingh R, Hedges Team. Developing optimal search strategies for detecting sound clinical prediction studies in MEDLINE. AMIA Annu Symp Proc. 2003:728-32.

17. Wilczynski NL, Haynes RB. Developing optimal search strategies for detecting clinically sound causation studies in MEDLINE. AMIA Annu Symp Proc. 2003:719-23.

18. Haynes RB, Wilczynski NL. Optimal search strategies for retrieving scientifically strong studies of diagnosis from Medline: analytical survey. BMJ 2004 May 1;328(7447): 1040. doi:10.1136/bmj.38068.557998.EE.

19. Wilczynski NL, Haynes RB. Developing optimal search strategies for detecting clinically sound prognostic studies in MEDLINE: an analytic survey. BMC Med. 2004 Jun 9;2:23.

20. Wong SS, Wilczynski NL, Haynes RB, Hedges Team. Developing optimal search strategies for detecting clinically relevant qualitative studies in MEDLINE. Stud Health Technol Inform 2004;107(Pt 1):311-6.

21. Wilczynski NL, Haynes RB, Lavis JN, Ramkissoonsingh R, Arnold-Oatley AE, HSR Hedges team. Optimal search strategies for detecting health services research studies in MEDLINE. CMAJ. 2004 Nov 9;171(10):1179-85.

22. Montori VM, Wilczynski NL, Morgan D, Haynes RB for the Hedges Team. Optimal search strategies for retrieving systematic reviews from MEDLINE: an analytical survey. BMJ. 2005 Jan 8;330(7482):68. doi:10.1136/bmj.38336.804167.47.

23. Haynes RB, McKibbon KA, Wilczynski NL, Walter SD, Werre SR, Hedges Team. Optimal search strategies for retrieving scientifically strong studies of treatment from Medline: analytical survey. BMJ. 2005 May 21;330(7501): 1179. doi:10.1136/bmj.38068.557998.EE.

24. U.S National Library of Medicine/National Institutes of Health. PubMed Subject Subsets [Internet]. May 2009. [rev. 18 May 2009; cited 8 Sep 2009]. Available from: http://www. nlm.nih.gov/bsd/pubmed_subsets.html.

25. Ovid Technologies Inc. Ovid MEDLINE ${ }^{\circledR}$ Database Guide [Internet]. Jan 2008. [rev. 9 Jan 2008; cited 8 Sep 2009]. Available from: http://www.ovid.com/site/products/ovidguide/ medline.htm\#stopwords.

26. Shojania KG, Bero LA. Taking advantage of the explosion of systematic reviews: an efficient MEDLINE search strategy. Eff Clin Pract. 2001 Jul-Aug;4(4):157-62.

27. NCBI. PubMed Clinical Queries Table [Internet]. [cited 8 Sep 2009]. Available from: http://www.ncbi.nlm.nih.gov/corehtml/ query/static/clinicaltable.html.

-28. Haynes RB, Walker CJ, McKibbon KA, Johnston ME, Willan AR. Performances of 27 MEDLINE systems tested by searches with clinical questions. J Am Med Inform Assoc. 1994;1(3):285-295. doi:10.1136/jamia.1994.95236159.

29. Walker CJ, McKibbon KA, Haynes RB, Johnston ME. Performance appraisal of online MEDLINE access routes. Proc Annu Symp Comput Appl Med Care. 1992;483-7.

30. Irvin E. Does it matter which version of MEDLINE you search? [abstract]. XII Cochrane Colloquium, Ottawa, Canada, October 2-6 2004;140. [cited 8 Sep 2009]. Available from: http://www.mrw.interscience.wiley.com/cochrane/clcmr/ articles/CMR-6682/frame.html. 
31. Elsevier Publishers. EMBASE: Excerpta Medica Database Guide [Internet]. [rev 21 Aug 2007; cited 8 Sep 2009]. Available from: http://www.ovid.com/site/products/ovidguide/ embase.htm.

32. US National Library of Medicine. NCBI Help Manual, PubMed Help. Text Words [Internet]. [rev 9 Jul 2009; cited 8 Sep 2009]. Available from: http://www.ncbi.nlm.nih.gov/ bookshelf/br.fcgi?book=helppubmed $\&$ part $=$ pubmedhelp \# pubmedhelp.Text_Words_TW.

33. US National Library of Medicine. NCBI Help Manual, PubMed Help. Stopwords [Internet]. [rev 9 Jul 2009; cited 8 Sep 2009]. Available from: http://www.ncbi.nlm.nih.gov/ bookshelf/br.fcgi?book=helppubmed $\&$ part $=$ pubmedhelp\& rendertype $=$ table $\&$ id $=$ pubmedhelp.T43.

34. Port T. Proximity Search Review [Internet]. NLM Tech Bull. 1996 Jan-Apr;(288-289), 16. [cited 8 Sep 2009]. Available from: ftp://nlmpubs.nlm.nih.gov/nlminfo/newsletters/techbull/ pdf_tb/janapr96.pdf.

35. Bakker, S and Brand, D. 2008 Embase/Ovid \& Embase.com versus PubMed \& Medline/Ovid: Some comparisons [Internet]. 11th EAHIL Conference. [rev Jun 2008; cited 8 Sep
2009]. Available from: http://www.terkko.helsinki.fi/bmf/ EAHILppt/Dieuwke_Brand.pdf.

36. Cook DJ, Mulrow CD, Haynes RB. Systematic reviews: synthesis of best evidence for clinical decisions. Ann Intern Med. 1997 Mar 1;126(5):376-80.

37. Health Information Research Unit, McMaster University. Search strategies for MEDLINE in Ovid syntax and the PubMed translation [Internet]. June 2007. [rev. 29 Jun 2007; cited 8 Sep 2009]. Available from: http://hiru.mcmaster.ca/ hiru/HIRU_Hedges_MEDLINE_Strategies.aspx.

38. NCBI. PubMed Clinical Queries [Internet]. [Cited 8 Aug 2009]. Available from: http://www.ncbi.nlm.nih.gov/corehtml/ query/static/clinical.shtml.

39. US National Library of Medicine. MEDLINE/PubMed Resources. Special Queries [Internet]. [Cited 8 Aug 2009]. Available from: http://www.nlm.nih.gov/bsd/special_queries.html.

40. Bradley SM. Examination of the Clinical Queries and Systematic Review 'Hedges' in OvidSP EMBASE and three MEDLINE Interfaces (OvidSP, PubMed and EBSCO). UBC LIBR594: Directed Studies Report. 16 Aug 2009; rev. 4 Sep 2009. 
This article has been cited by:

1. Bradley Susan M.. 2011. LETTER / LETTRE. Journal of the Canadian Health Libraries Association 32:3, 177-179. [Citation] [Full Text] [PDF] [PDF Plus]

2. Wilczynski Nancy L., Brian Haynes R., Ann McKibbon K.. 2011. LETTER / LETTRE. Journal of the Canadian Health Libraries Association 32:3, 181-182. [Citation] [Full Text] [PDF] [PDF Plus]

3. 2011. Full issue in PDF / Numéro complet en form PDF. Journal of the Canadian Health Libraries Association 32:3, 133-184. [Citation] [PDF] [PDF Plus] 